\title{
Concurrent Scanning for High Quality Hand Over Support in Mobile WiMAX Networks
}

\author{
${ }^{1}$ ARATHI R SHANKAR, ${ }^{2}$ PRABHU RADDY, ${ }^{3} \mathrm{~V}$. SAMBASIVA RAO \\ ${ }^{1}$ Associate Professor, Department of Electronics and Communication Engineering, \\ BMS College of Engineering Bangalore, Karnataka-560019, India \\ arathi.rshekhar@gmail.com \\ ${ }^{2}$ Department of Electronics and Communication Engineering, \\ BMS College of Engineering Bangalore, Karnataka-560019, India \\ ptreddyc@gmail.com \\ ${ }^{3}$ Professor, Department of Electronics and Communication Engineering, \\ PES Institute of Technology Bangalore, Karnataka-560019, India \\ vsrao@pes.edu
}

\section{ABSTRACT}

Mobile WiMAX is IEEE 802.16e standard established for mobile broadband wireless access (BWA). Mobility in WiMAX system is an important issue when the mobile station (MS) moves and be handover between base stations (BSs). This issue causes an unnecessary neighboring BS scanning and association, handover delay, and MAC overhead which may affect real-time applications.

The MS movement direction prediction (MMDP) based MS scanning is used to reduce the number of scanning's required in mobile WiMAX handover process. In this paper with reduction in number of scanning's we propose to reduce the scanning delay required for evaluated two best target BSs by using concurrent scanning process. Network issues like ideal sectors in WiMAX 2 (IEEE 802.16m), network congestion and fast change in RSS are important parameters which can affect the QoS. With this proposed model we are able to provide the high quality hand over support in mobile WiMAX wherein we are able to solve the above stated network problems. Here on using the concurrent scanning process the authors are able to reduce the scanning delay by $40 \%$. As a part of this paper, network layer parameters called routing protocols are analyzed for hand over support. This analysis shows an efficient routing protocol for real time application and the best effort service. This reviles that fisheye protocol is better to use for smaller network and LANMAR for the larger networks in real time application and IERP protocol is good for best effort services.

\section{KEYWORDS}

Mobile WiMAX, Scanning, MS movement direction prediction (MMDP), serving base station (SBS), target base station (TBS), Average End to End Delay, Average Data Received.

\section{SUBJECT CLASSIFICATION}

Wireless Networking, Computer Networking and Mobile Communication.

\section{TYPE (METHOD/APPROACH)}

In this work, network simulator QualNet 6.1 has been used to evaluate the performance of Mobile WiMAX with various routing protocols. MATLAB 7.10 has been used to simulate the mobile WiMAX network for high quality hand over support.

\section{Council for Innovative Research}

\author{
Peer Review Research Publishing System
}

\section{JOURnal: INTERNATIONAL JOURNAL OF COMPUTERS \& TECHNOLOGY}

\author{
Vol 10, No 2 \\ editor@cirworld.com \\ www.cirworld.com, member.cirworld.com
}




\section{INTRODUCTION}

WiMAX is an acronym for worldwide interoperability for Microwave Access. It is a broadband wireless access (BWA) technology which provides an easy, time saving and low cost deployment method for the next generation (4G) infrastructure. It provides broadband access in rural and urban areas over fixed wireless channels. Mobile WiMAX is IEEE 802.16e standard established for mobile BWA. Data, Voice over Internet Protocol and video streaming are the main traffic from the users. In day today life the demand for high speed transmission data rate is growing tremendously. Research for new and more advance communication system is vital to fulfill the world demand. Therefore, the introduction of high speed network system providing high quality of network is one of the possible solutions at this moment.

\subsection{Mobile WiMAX}

There are lots of challenges involved in the service provided by the Mobile WiMAX. Because of its wireless nature, it can be faster to deploy, easier to scale and more flexible, thereby giving it the potential to serve customers not served or not satisfied by their wired broadband alternatives. Some Mobile WiMAX as IEEE 802.16e supports mobility and is also capable to provide fixed access [2]. Characteristics of IEEE 802.16e are Advanced antenna diversity schemes, HARQ, adaptive antenna system(AAS),MIMO technology, thereby improving indoor penetration, introducing turbo coding and low density parity check (LDPC), introducing downlink sub-channelization, allowing administrators to trade coverage for capacity or vice versa, adding an extra QoS class for VoIP applications [3]. . The deployment of Mobile WiMAX network topology is as shown in figure 1.

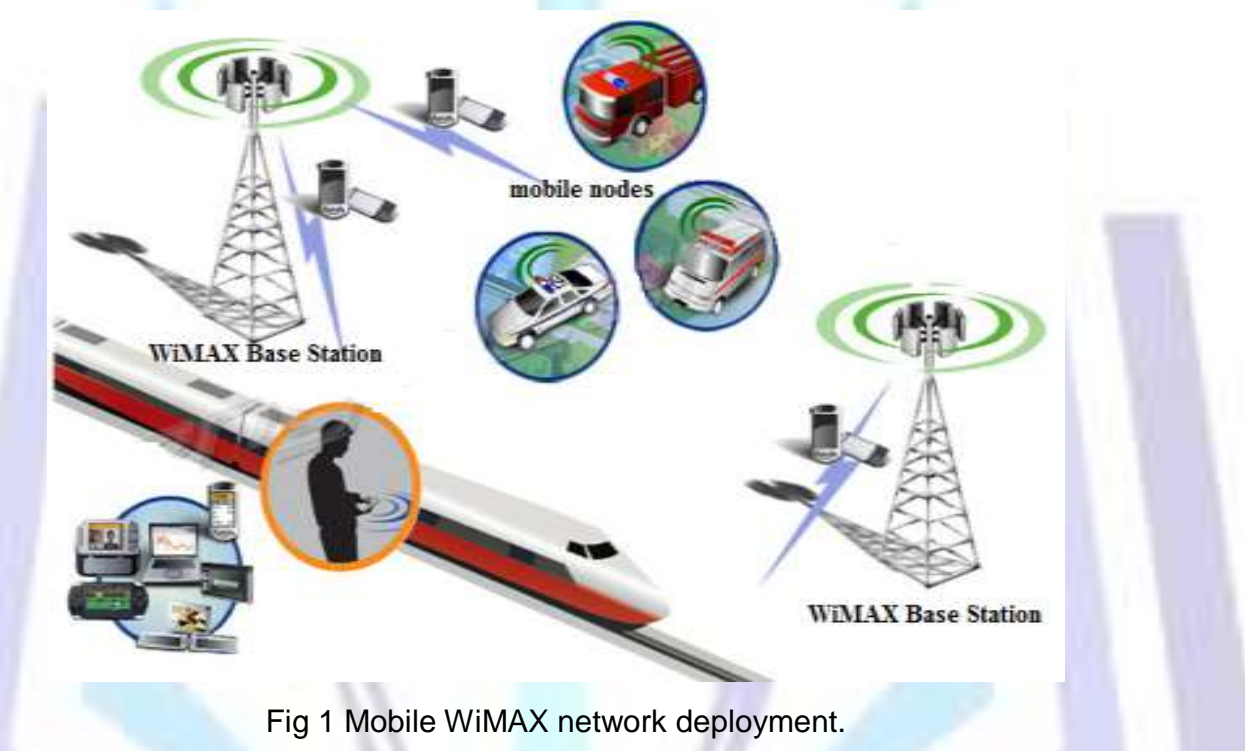

Although the basic handover procedure has been introduced for IEEE 802.16e to support full mobility, the emerging standards of IEEE $802.16 \mathrm{j}$ and IEEE $802.16 \mathrm{~m}$ are also exploiting the same handover principles of IEEE $802.16 \mathrm{e}$ with some other amendments regarding to the requirements of the new standard [4]. the most recent standard of IEEE $802.16 \mathrm{~m}$ promises to meet the IMT-Advanced requirements which to provide high data rates of at least $1 \mathrm{Gbps}$ for the fixed subscriber and $100 \mathrm{Mbps}$ for mobile subscriber at a vehicular speed of up to $350 \mathrm{~km} / \mathrm{h}$. Also, the IEEE $802.16 \mathrm{~m}$ supports the MAC and PHY features of the location based service (LBS) solution, where the BS can track the MS movement [5].

\subsection{Handover}

Handover mechanism is one of the critical operations in mobile WiMAX. The handover mechanism handles mobile station (MS) for switching from one serving base station (SBS) to target base station (TBS). This can affects the QoS \& capacity of mobile broadband network. QoS is the performance level of the services offered by the network to the user. It is required for efficient utilization of resources and for better information delivery.

The basic handover procedure can be categorized into two main phases; the network topology acquisition phase (NTAP) or pre-handover phase, and the actual handover phase (AHOP)[1]. The first phase includes the network topology advertisement, neighbouring BS scanning, and association. The second phase consists of cell selection, handover decision and initiation, and network re-entry including ranging, authorization and re-registration.

Hand over take place in two ways: Mobility between subnets in the same network (micro mobility) \& between two different network domains. The hand over techniques in the micro mobility can be divided into soft hand over (make_ before break) \& hard hand over (break_before_make). In Soft hand over connection with the serving base station is terminated before a mobile station switches to another base station. It will have zero break time during hand over at the cost of lower spectral efficiency. Hard hand over $(\mathrm{HHO})$ uses a break before makes approach. A typical mobile WiMAX network uses packet switching with mostly bursty delay tolerant data traffic. Therefore Hard HO is used in mobile WiMAX. 


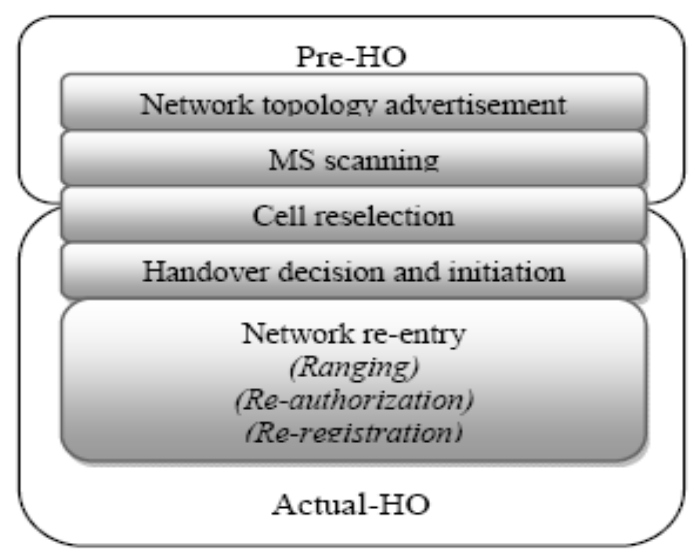

Fig.2 Hand over phases

\subsection{Scanning}

MS Scanning is the process where the MS must synchronize with all advertised neighbour BSs (nBS) to select the best BS candidate for the incoming handover action. Without terminating the connection between the SBS and MS, the SBS will schedule the scanning intervals or sleep-intervals to MS for scanning. Due to the inefficient factor of the redundant scanning activities in this phase, all the data transmission will be paused, and it may cause handover delay and throughput degradation. This issue will affect the real-time continuity of multimedia application. Authors in [7] proposed MS movement direction prediction (MMDP) based MS scanning scheme for mobile WiMAX system. Through the WiMAX's backbone and LBS features, the SBS can know the MS and its nBSs locations. Based on the cell sectoring-zoning, and the past and current MS position, the SBS can predict the MS direction which will be described in details. After the MS receives the MOB_NBR-ADV massage and the receiving signal quality reaches the scanning threshold, the MS will request a scanning interval with a list of the $\mathrm{nBSs}$. The SBS will responses the scanning requests by sending SCN_RSP message which include a shortlist of the nBSs (two BSs to be scanned). After scanning, the SBS will choose the best TBS among the scanned base stations. Due to the inefficient factor of the redundant scanning activities in this phase, all the data transmission will be paused, and it may cause handover delay and throughput degradation. This issue will affect the real-time continuity of multimedia application sessions. Hence pre-handover delay minimization is the important issue; Fig (2) shows the pre-hand over phase. SBS sends out the HO-ADV signal to MS in response to this it will send the HO-REQ to SBS to indicate the hand over request, which intern sends the HO-RSP signal for the other nBS scanning [6]. After scanning for $\mathrm{n}$ base stations actual traffic is allowed to access the network.

The rest of this paper is organized as follows. Section 2 provides technical background of Mobile WiMAX HO methodology using MMDP Scheme. In section 3, we introduced QualNet Network scenario and proposed concurrent scanning method. Then in section 4, the simulation results are evaluated. Finally, in section 5, a summary and conclusions based on simulations performed are presented.

\section{MMDP SCHEME}

The Serving Base Station keeps track of the MS movement location and trajectory. Based on [5], the BS coverage area is divided into three zones as no handover (No-HO) zone, low handover (Lo-HO) zone, high hand over (High-HO) zone based on signal quality and six sectors as shown in Figure (3). The No-HO zone is having least handover probability, while Lo-HO and High-HO zones are having low and high handover probabilities. This division subsequently reduces the actual area of MS's random movement tracking during a potential handover activity [8].

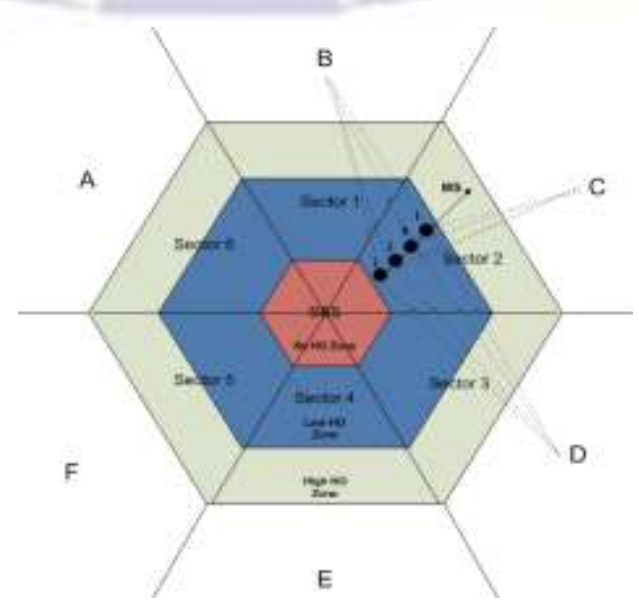

Fig 3 MMDP Scheme 
Unlike [1], the SBS will utilize this information to calculate the distance between MS and only to the nBSs which located in the same sector as the current MS location where the neighboring sectors will be considered on distance calculation process. As an example of MS in Figure 3 where only nBSs B, Cand D are considered, the SBS in the Lo-HO zone will start to predict the MS movement direction The SBS calculates the distance between the MS and three potential TBSs of B, C and D based on coordinate distance formula which is derived as follows:

$$
d=\operatorname{sqrt}\left(\left(x_{2}-x_{1}\right)^{2}+\left(y_{2}-y_{1}\right)^{2}\right)
$$

Depending on their relative distance with MS, only the BS B and C are considered as a target base station. Now there is a race between $B$ and $C$ to become serving base station [9]. In this way we can reduce the number of scanning's in the pre hand over process.

\section{PROPOSED MODEL}

The proposed model for high quality hand over support is divided into two simulations as QualNet simulation and MATLAB simulation. QualNet simulation is used to evaluate the efficient routing protocol for high Quality hand over support in mobile WiMAX. MATLAB simulation is used to solve the network problems by using concurrent scanning approach.

\subsection{Efficient Routing Protocol for Mobile WiMAX}

WiMAX Scenario consisting of two base stations and four mobile stations with all the parameters as per the IEEE 802.16e standard is modeled using Qualnet 6.1 to analyze the best routing protocol for the Hand over support. The simulation parameters are as given in table 1 and the network scenario as shown in figure 4.

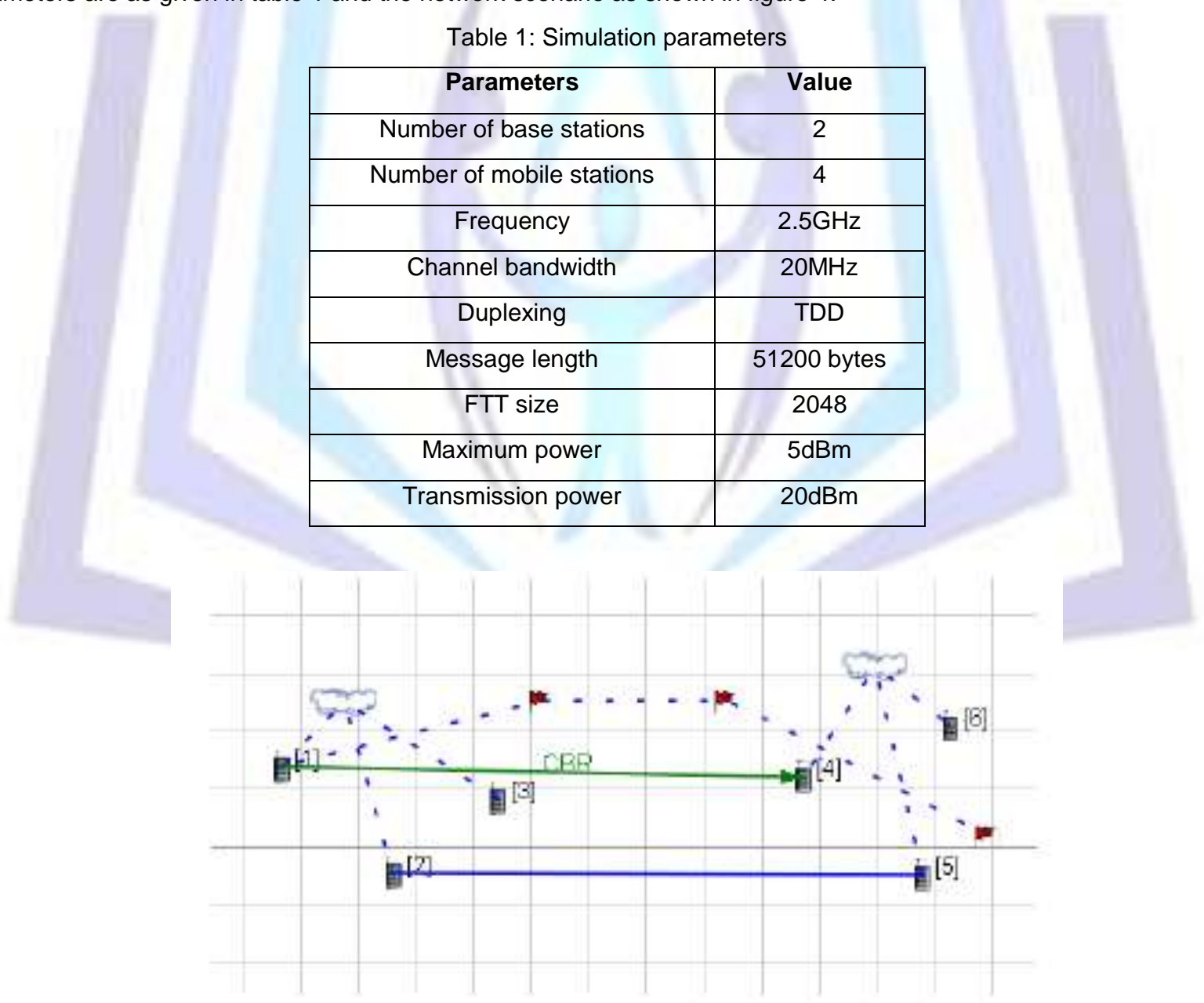

Fig 4 WiMAX simulation scenario

An analysis has been made using different routing protocols for the unicast data received and the corresponding delay is involved in delivering the message from source to destination. A small introduction to the routing protocol used in the simulation is given below. 


\subsubsection{AODV}

Ad hoc On-Demand Distance Vector (AODV) Routing is a routing protocol for mobile ad hoc networks (MANETs) and other wireless ad-hoc networks. It is a reactive routing protocol, meaning that it establishes a route to a destination only on demand. In contrast, the most common routing protocols of the Internet are proactive, meaning they find routing paths independently of the usage of the paths. The advantage of AODV is that it creates no extra traffic for communication along existing links. Also, distance vector routing is simple, and doesn't require much memory or calculation. However AODV requires more time to establish a connection, and the initial communication to establish a route is heavier than some other approach.

\subsubsection{BELLMAN-FORD}

The Bellman-Ford algorithm is an algorithm that computes shortest paths from a single source vertex to all of the other vertices in a weighted digraph. It is slower than Dijkstra's algorithm for the same problem, but more versatile, as it is capable of handling graphs in which some of the edge weights are negative numbers. In such a case, the Bellman-Ford algorithm can detect negative cycles and report their existence, but it cannot produce a correct "shortest path" answer if a negative cycle is reachable from the source, because there is no well-defined "shortest path" in such cases.

\subsubsection{DYMO}

The DYMO is an abbreviation for dynamic AODV. This routing protocol is successor to the popular Ad hoc On-Demand Distance Vector (AODV) routing protocol and shares many of its benefits. It is, however, slightly easier to implement and designed with future enhancements in mind. This can work as both a pro-active and as a reactive routing protocol, i.e. routes can be discovered just when they are needed.

\subsubsection{FSR}

Fisheye State Routing (FSR) belongs to the class of proactive (table-driven) ad hoc routing protocols and its mechanisms are based on the Link State Routing protocol used in wired networks. It tries to minimize the routing overhead by using a fisheye technique. Each node assigns other network participants to specific fisheye scopes dependent on their distance to the node itself. The amount of routing information is reduced by assuming longer link-state update intervals for nodes at higher distances than for network participants in the node's vicinity. Thus, FSR is intended to scale well in large mobile ad hoc networks and supports high rates of mobility.

\subsubsection{IERP}

The Inter-zone Routing Protocol (IERP) is a reactive routing component of the Zone Routing Protocol (ZRP). IERP adapts existing reactive routing protocol implementations to take advantage of the known topology of each node's surrounding $R$ hop neighborhood (routing zone).When a global route discovery is required, the routing zone based broadcast service can be used to efficiently guide route queries outward, rather than blindly relaying queries from neighbor to neighbor. Once a route has been discovered, IERP can use routing zones to automatically redirect data around failed links.

\subsubsection{LANMAR}

LANMAR combines the features of Fisheye State Routing (FSR) and Landmark routing. This routing protocol is used for Large Scale Wireless Ad Hoc Networks with Group Mobility. It provides efficient and scalable routing in large, mobile, ad hoc environments in which group mobility applies. The Landmark Ad-hoc Routing Protocol (LANMAR) is designed to dramatically reduce routing table size and routing update overhead in large-scale ad-hoc networks that exhibit group mobility.

\subsection{Proposed Scanning Process}

In order to accomplish concurrency the program is divided into many processes. We know that processor speed ( $\mu \mathrm{s})$ is more comparing to the $\mathrm{I} / \mathrm{O}$ processing $(\mathrm{ms})$ as shown in figure 5 . According to concurrent scanning process once the $\mathrm{I} / \mathrm{O}$ request comes in the process flow is given to the next process till the serving process gets the i/o information.

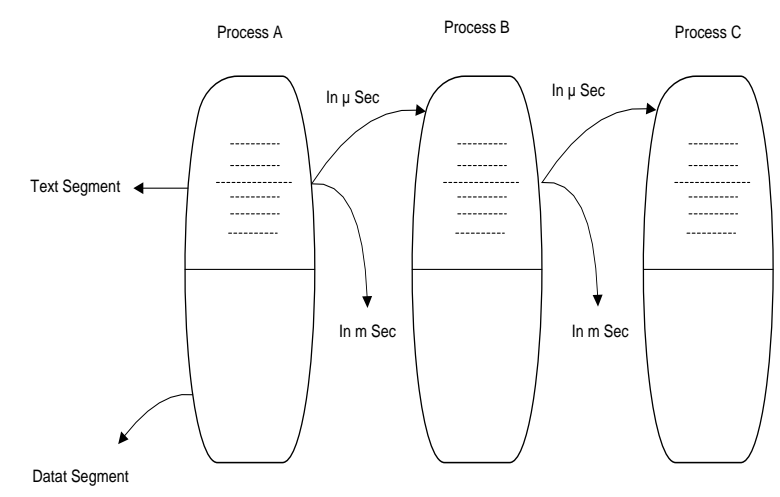

Fig 5 concurrent processing 
After detecting the two best possible targets base stations we consider the concurrent scanning for both the TBSs. On doing this we proposed to reduce the time duration required for scanning. Hence we are able to achieve the reduced pre hand over delay. Concurrent scanning simulation is done on the MATLAB. The simulation follows the flow chart shown in figure 6 .

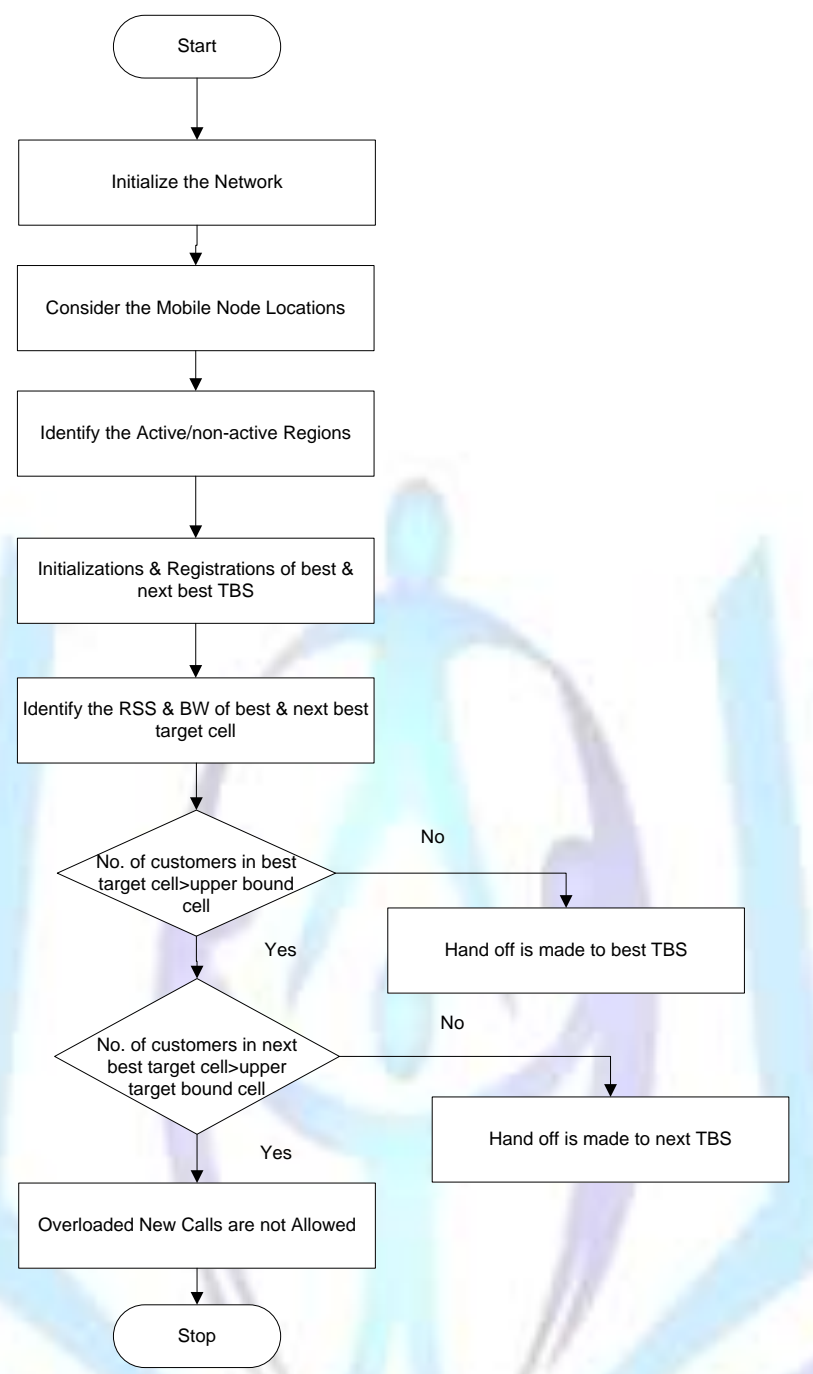

Fig 6 proposed flow chart

Bandwidth of each cell can be derived as follows:

$$
\mathrm{BR}_{\mathrm{B}}=\mathrm{C} \cdot \mathrm{P}_{\mathrm{B}} \cdot \mathrm{BW}_{\mathrm{MH} *} \frac{1}{\mathrm{D}_{\mathrm{B}}}
$$

where $\mathrm{C}$ is a constant.

In this work, we adopt the following mobility profile of the $\mathrm{MH}$ :

1. The speed of the $\mathrm{MH}$

2. The distance between the $\mathrm{MH}$ and the radius of base station

3. Probability to neighbor cell is larger if it is a hot cell.

Since the above-mentioned metrics are all time series, we can use them as the parameters of the grey system, and apply the predictive values of these parameters as the inputs to the following equation

\section{Boundary $=$ radius $x$ ratio $\times$ speed $\times$ direction}

where Radius is that of the base station, Ratio denotes the distance between the $\mathrm{MH}$ and the radius of base station, Speed represents the speed of the $\mathrm{MH}$, and Direction stands for the mobility towards the neighboring cells. 


\section{SIMULATION RESULTS}

\subsection{QualNet Simulation Results}

The amount of data sent by the transmitting node i.e. node 1 is 51200 bytes. The scenario is run with different routing protocols on the Qualnet environment and an observation is made on the amount of data being received in bytes and average end to end delay incurred for destination node i.e. node id 4 . The figures from 7 to 18 shows the simulation results for different routing protocols.

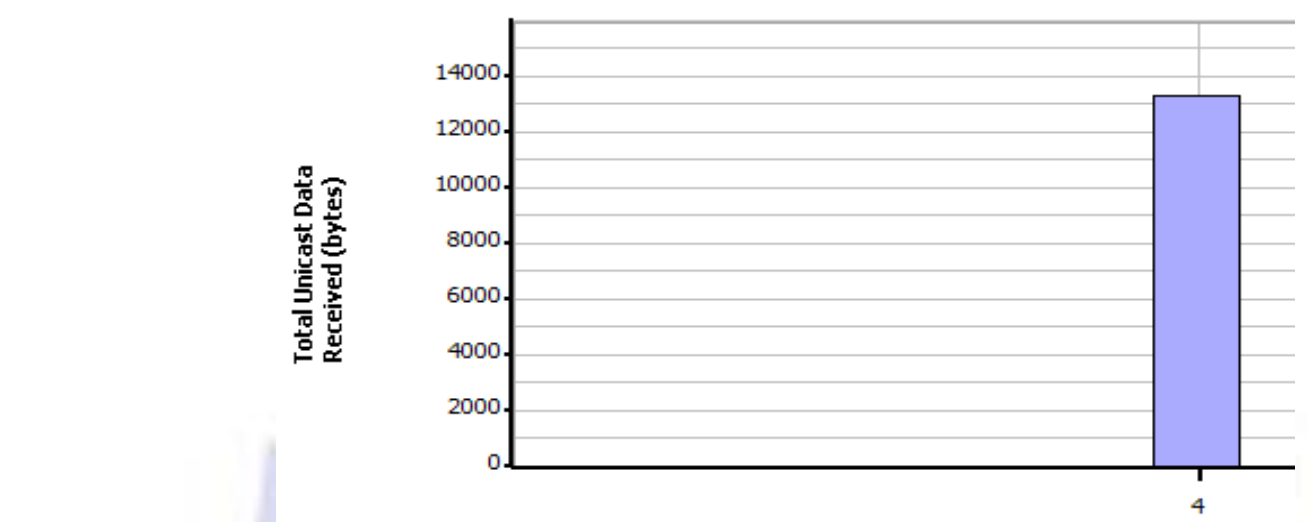

Fig 7 AODV received data13, 312 bytes

Fig 8 AODV end to end Delay $0.6246 \mathrm{sec}$
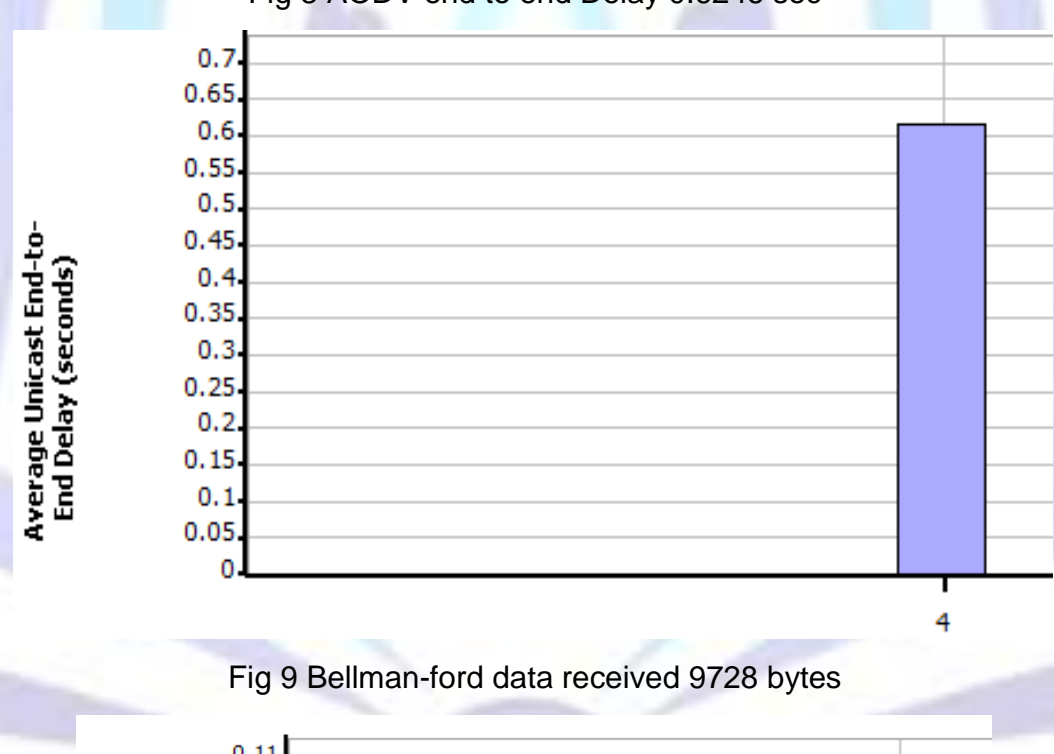

Fig 9 Bellman-ford data received 9728 bytes

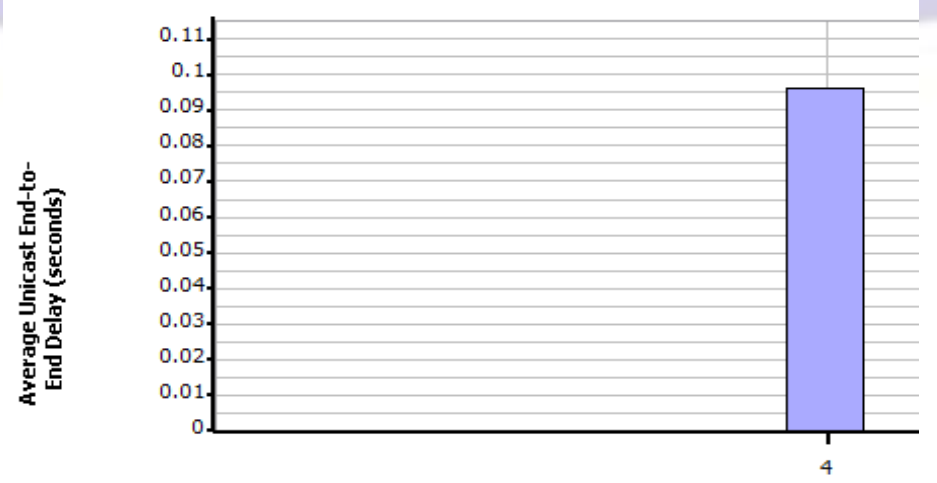

Fig 10 Bellman-ford Delay $0.09 \mathrm{sec}$ 


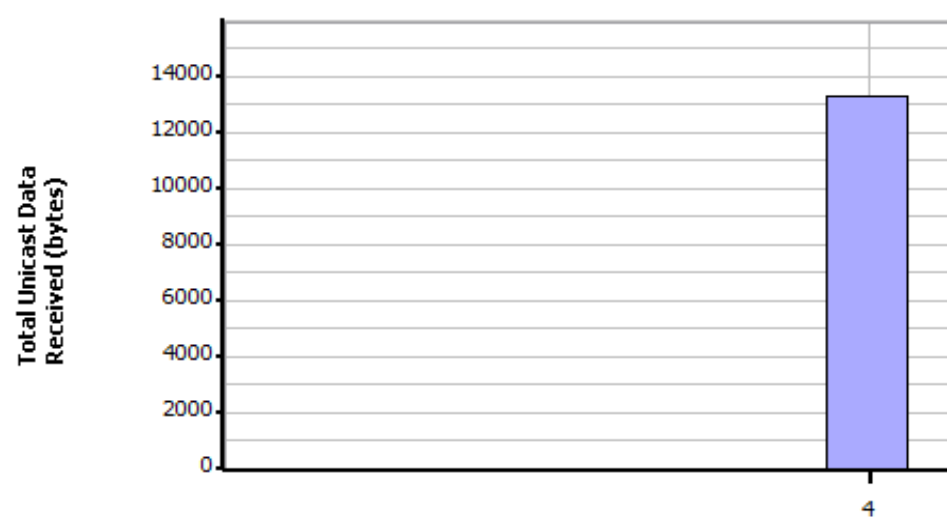

Fig 11 DYMO data received 13,312 bytes

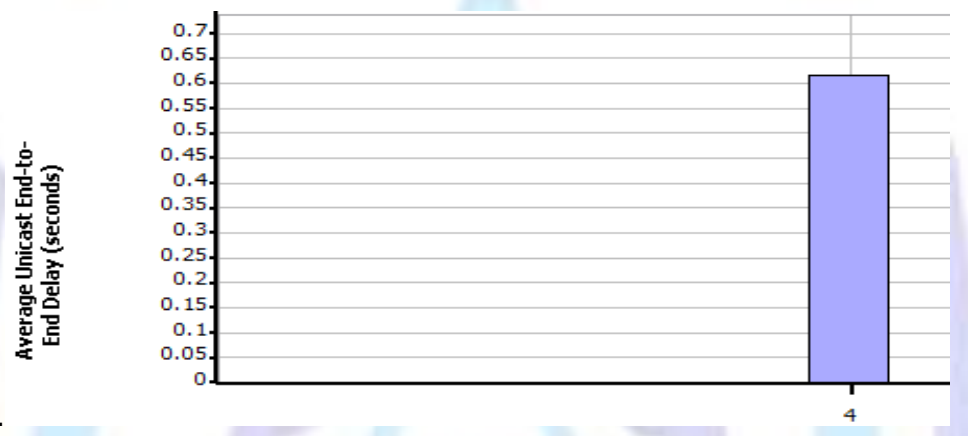

Fig 12 DYMO end to end Delay $0.6169 \mathrm{sec}$

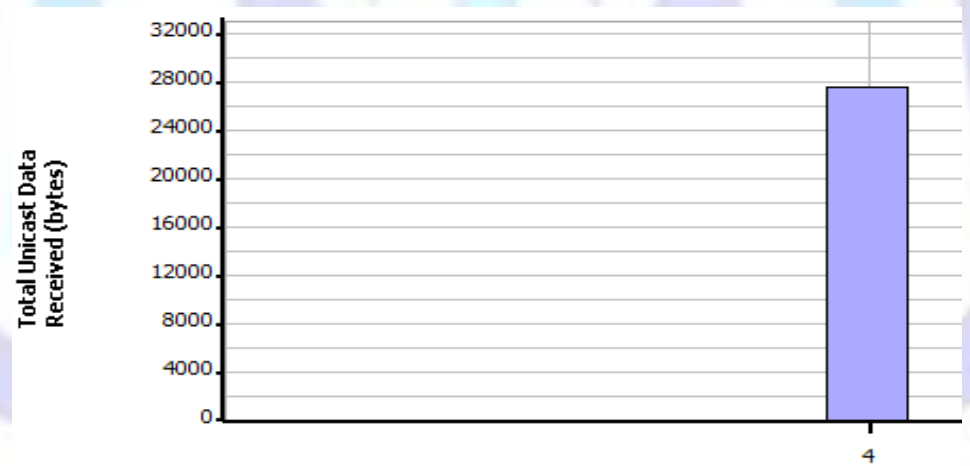

Fig 13 Fisheye data received 27,648 bytes

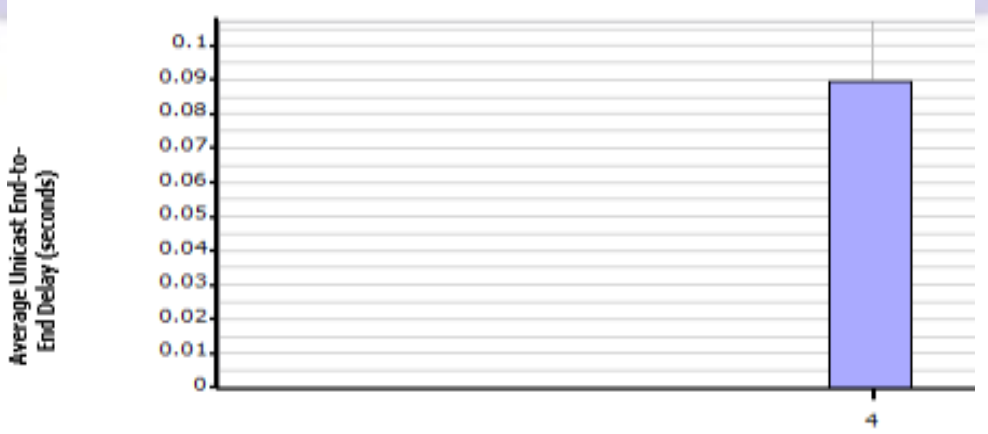

Fig 14 Fisheye end to end Delay $0.0896 \mathrm{sec}$ 


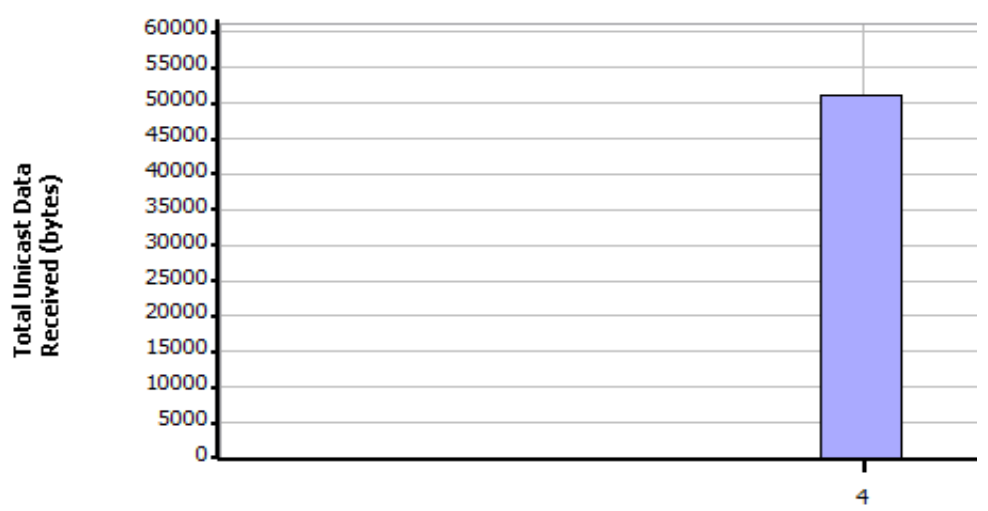

Fig 15 IERP data received 51200 bytes

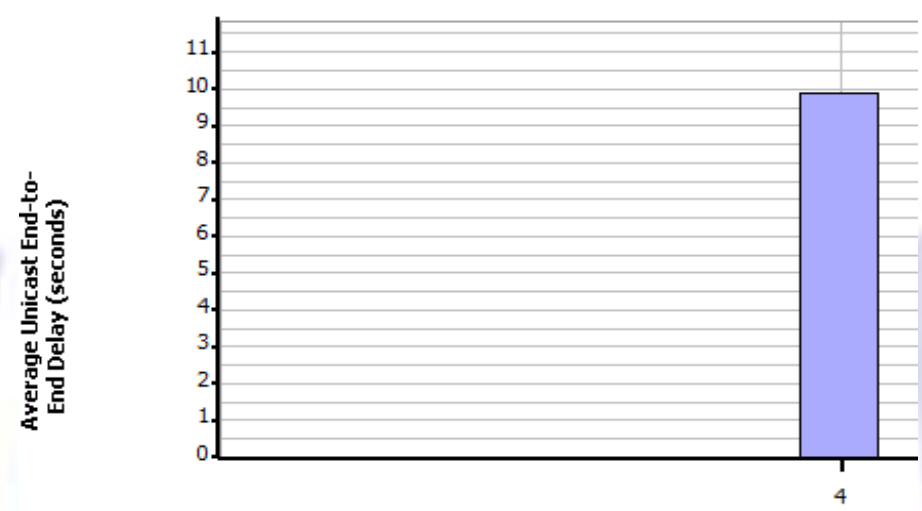

Fig 16 IERP end to end Delay $9.9024 \mathrm{sec}$

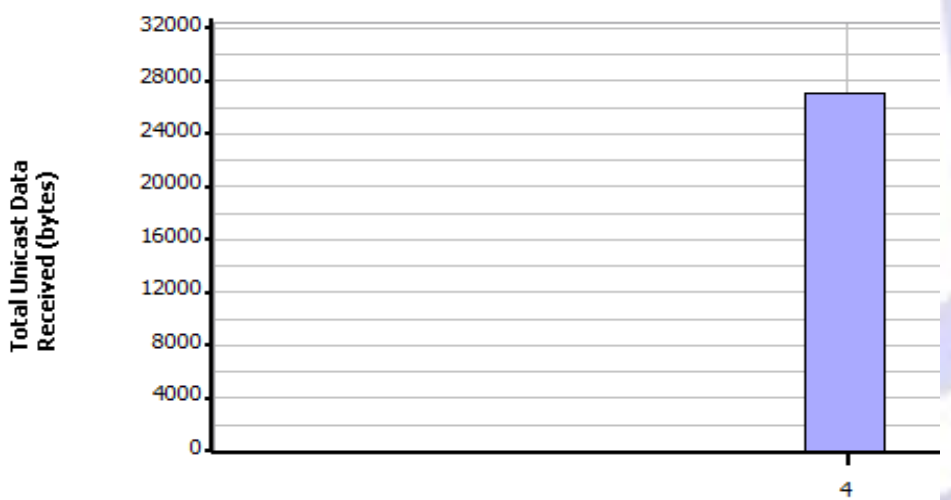

Fig 17 LANMAR data received 27,136

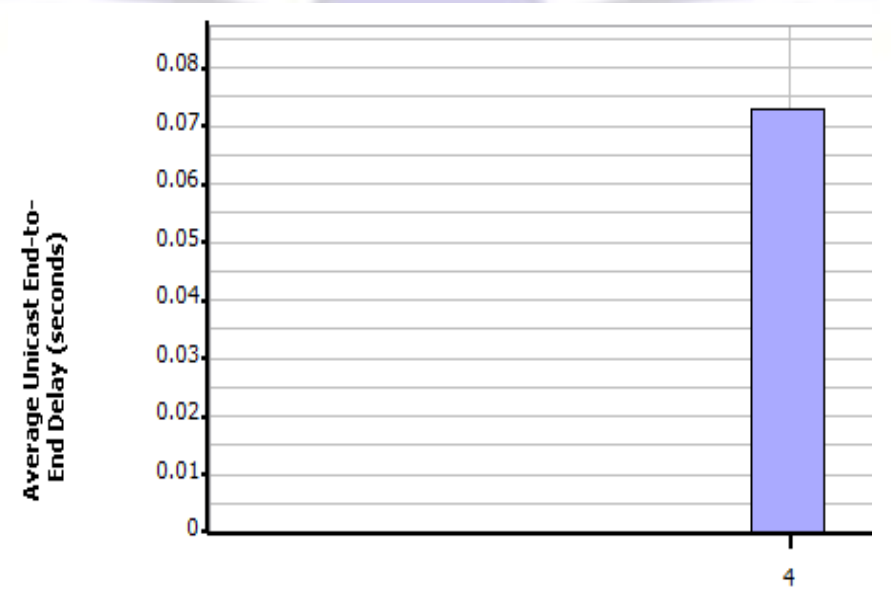

Fig 18 LANMAR end to end delay $0.073206 \mathrm{sec}$ 
The table 2 depicts that LANMAR is the best routing protocol for real time applications i.e. rtPS [13] service class and IERP has been found to be best suited for Best Effort service class. After on conducting the similar experiments for varying network sizes, it is observed that Fisheye protocol is optimal for smaller network and LANMAR is for the larger networks.

Table 2 Comparative table for routing protocols

\begin{tabular}{|l|l|ll|}
\hline ROUTING PROTOCOL & $\begin{array}{l}\text { UNICASTE DATA RECEIVED } \\
\text { (Bytes) }\end{array}$ & $\begin{array}{l}\text { END TO END DELAY } \\
(\mathrm{Sec})\end{array}$ & \\
\hline AODV & 13,312 & 0.624 & \\
\hline BELLMANN FORD & 9,728 & 0.09631 & \\
\hline DYMO & 13,312 & 0.6169 & \\
\hline FISHEYE & 27,648 & 0.0896 & \\
\hline IERP & 51,200 & 9.9024 & \\
\hline LANMAR & 27,136 & 0.0732 & \\
\hline
\end{tabular}

\subsection{MATLAB Simulation Results}

A WiMAX Scenario consisting of seven base stations out of which one is service base station and six are considered as target base stations. The green star represents the selected mobile node for the handover process, a blue star represents the corresponding base station of the cell and red stars are the serving mobile nodes in the cell. The location of mobile node requiring hand off between SBS to TBS is computed, if the mobile node is found to be inside the no hand off region as in figure 19 and hence hand off is not possible as given in figure 20 else hand off is possible. Depending on the handover possibilities we analyze three cases as described below.

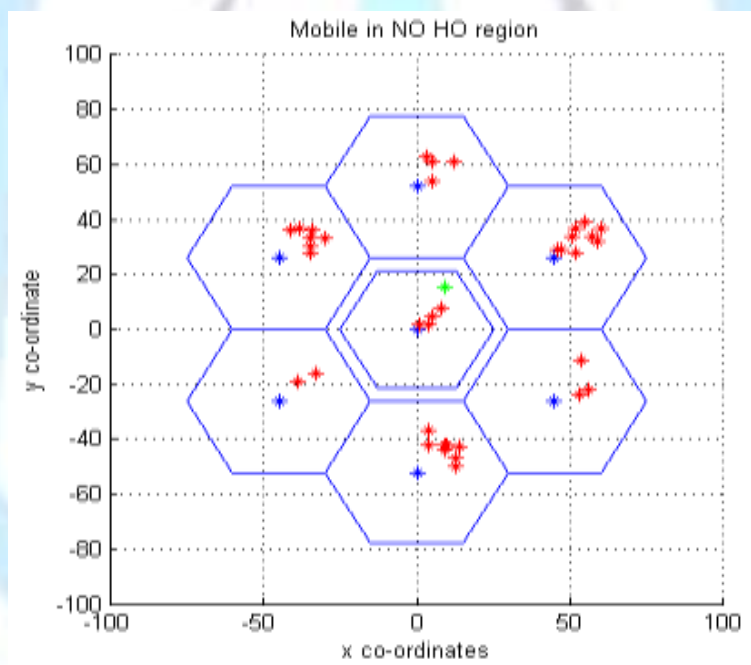

Fig 19 Mobile inside NO HO region

\section{Current MH - Speed}

Speed $=51.7846 \quad 99.4243 \quad 85.4852 \quad 96.2404$

Current MH - prob = $67.8941 \quad 40.3501 \quad 93.4979 \quad 47.9485$

Current $\mathrm{MH}$ - Distance

Dist $=23.1792 \quad 39.6290 \quad 70.5077 \quad 55.8559$

scaleFact $1=10$

Hand Off $=0$

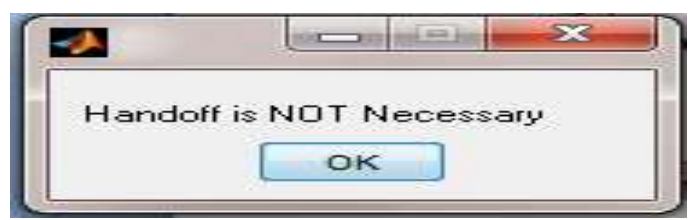

Fig 20 Hand off not necessary

\subsubsection{NORMAL BEST TARGET CELL}


Normal target cell is one in which numbers of customers are less than the upper bound limit. Each cell consists of a minimum and maximum number of users denoted by lower bound and upper bound numbers. In this case the number of users less than the upper bound limit as shown in figure 21 hence handover is possible with best target station as shown in figure 23.

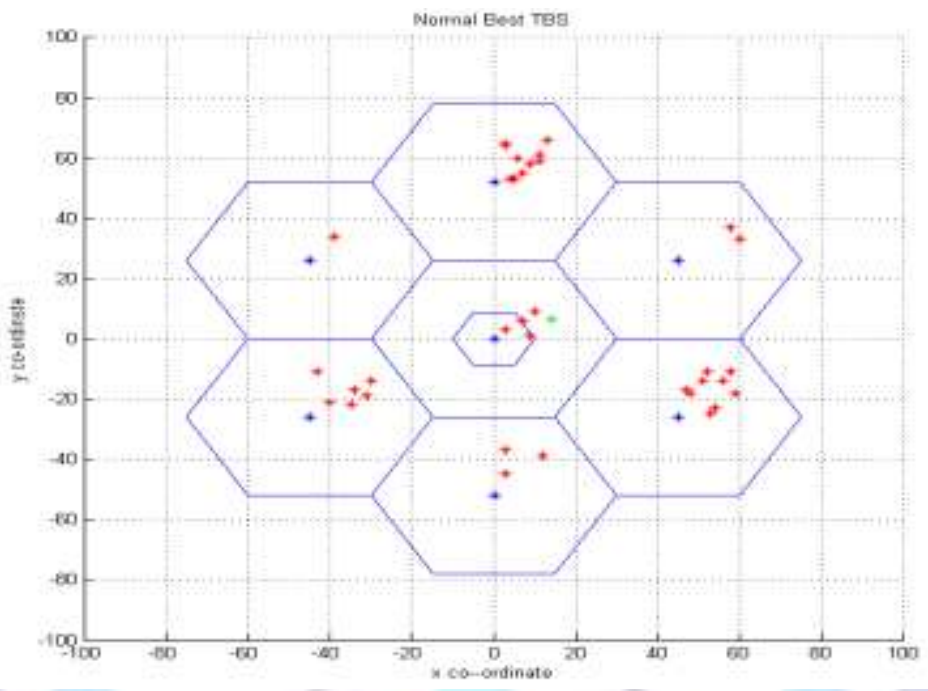

Fig 21 Normal best target cell

Network Initialization and Simulation parameters

Current MH - Speed

Speed $=81.4724 \quad 90.5792 \quad 12.6987 \quad 91.3376$

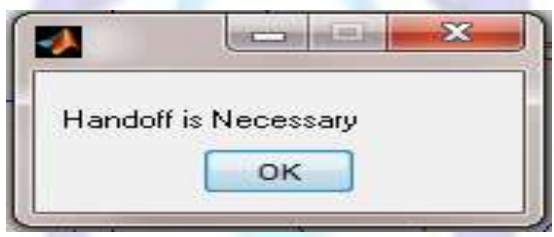

Fig 22 Handoff Necessary

Current MH - Probability

prob $=63.2359 \quad 9.7540 \quad 27.8498 \quad 54.6882$

Current MH - Distance

Dist $=95.7507 \quad 96.4889 \quad 15.7613 \quad 97.0593$

scaleFact1 $=10$

Hand Off $=1$

TBS Distance $=51.6655 \quad 65.2479 \quad 67.4699 \quad 57.0889 \quad 40.8801 \quad 37.0982$

Customer $=\begin{array}{lllllll}7 & 8 & 8 & 4 & 7 & 2\end{array}$

Best TBS $=6$

Next Best TBS $=5$

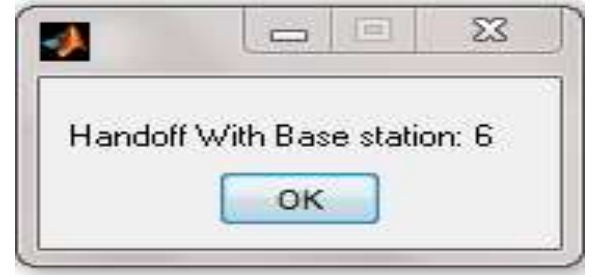

Fig 23 Handoff to Base Station 6 


\subsubsection{BOTH TBSS OVER LOADED}

In case both the selected target base stations are having customers more than upper bound is the over loaded TBSs case. The simulation result shown in figure 24 describes the above case. As the TBSs are over loaded with the customers there is no band width to allow the new incoming calls and hence the message is displayed as shown in figure 26.

Network Simulation

Current $\mathrm{MH}$ - Speed

speed $=75.0520 \quad 58.353355 .1793 \quad 58.3571$

Current $\mathrm{MH}$ - Probability

prob $=51.1820 \quad 8.2593 \quad 71.9570 \quad 99.6156$

Current $\mathrm{MH}$ - Distance

Dist $=35.4534 \quad 97.1259 \quad 34.6449 \quad 88.6544$

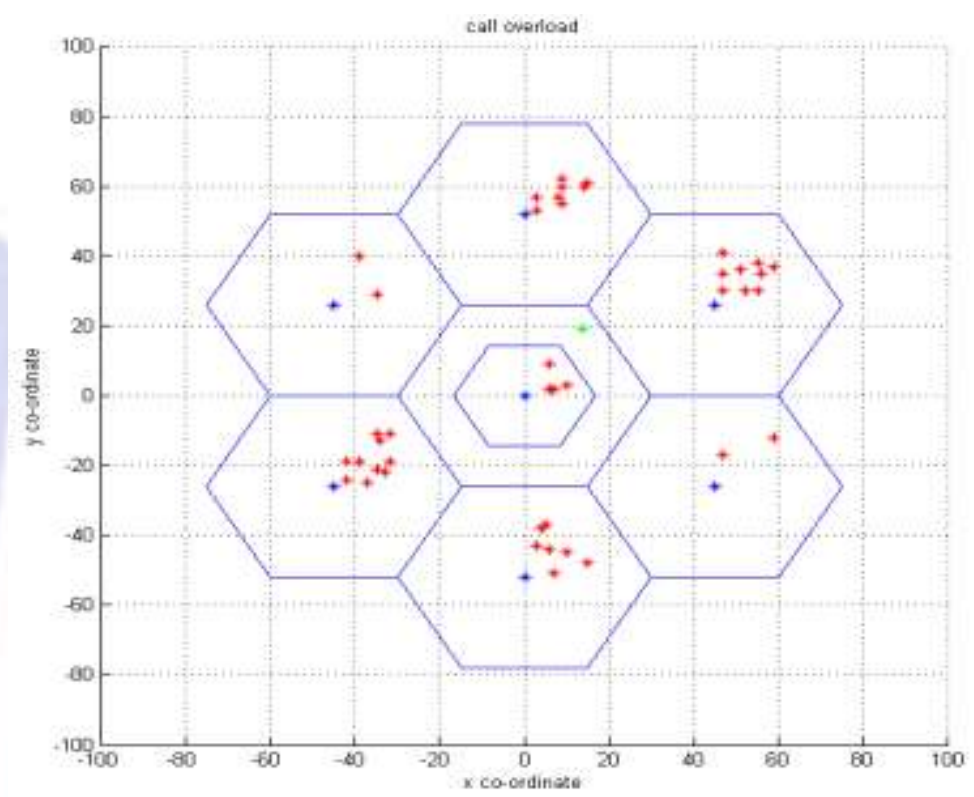

Fig 24 Both TBSs over loaded

scaleFact $1=10$

Handoff $=1$

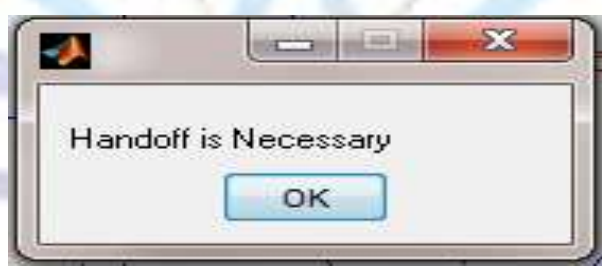

Fig 25 Hand off necessary

TBS Distance $=53.9143 \quad 67.6183 \quad 68.4552 \quad 55.9867 \quad 38.3280 \quad 36.8124$

Customer $=\begin{array}{llllll}10 & 9 & 3 & 6 & 10 & 8\end{array}$

Best TBS 6

Next Best TBS $=5$ 


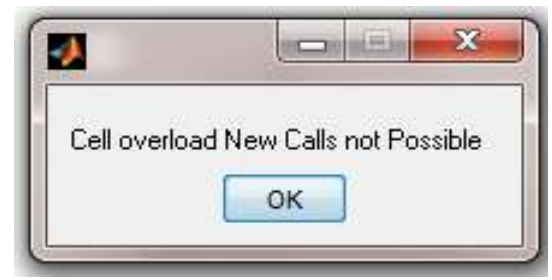

Fig 26 Call Dropping

\subsubsection{HAND OVER TO NEXT BEST TBS}

In this case best TBS is having more number of customers' compared to upper bound hence overloaded but next best TBS is having less number of customers' compared to upper bound hence it will allow the new incoming call. Here the hand off is given to next best TBS. network scenario is as shown in figure 27 and the message displayed is shown in figure 29.

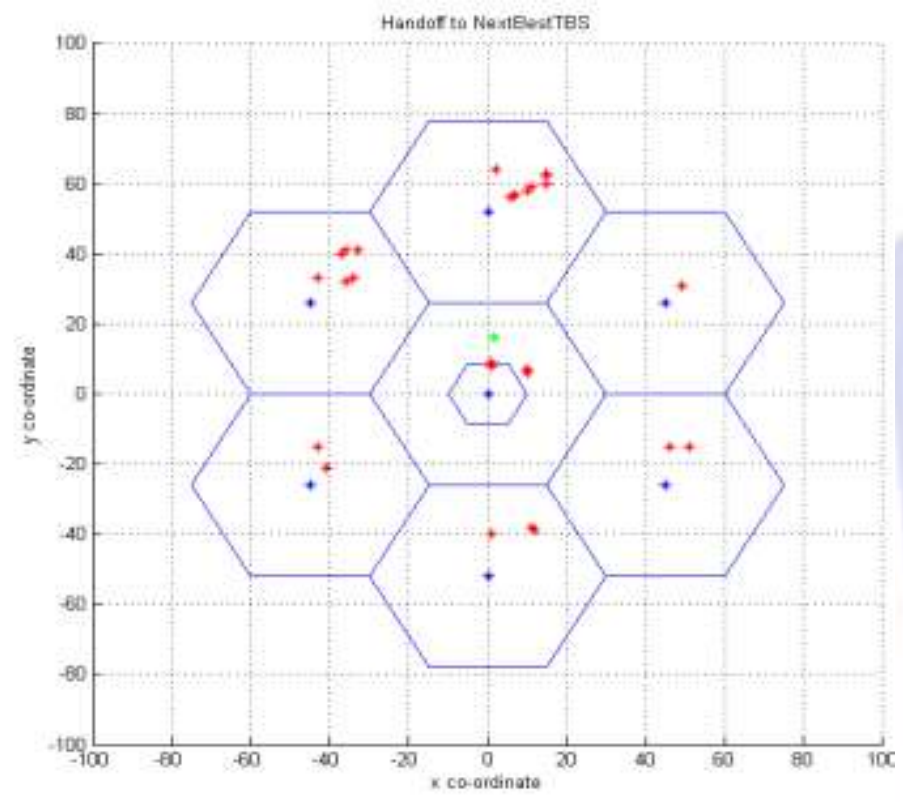

Fig 27 Hand over to next best TBS

Network Simulation

Current MH - Speed

Speed $=80.2416 \quad 66.9643 \quad 47.1603 \quad 94.4478$

Current $\mathrm{MH}$ - Probability

prob $=\begin{array}{llll}59.1481 & 22.6748 & 68.3844 & 32.0643\end{array}$

Current MH - Distance

Dist $=\begin{array}{llll}74.8919 & 99.8143 & 26.9733 & 93.1870\end{array}$

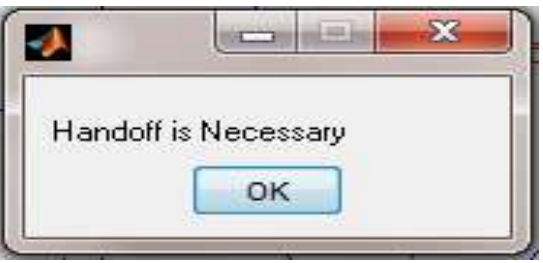

Fig 28 Handoff necessary

scaleFact $1=10$

Hand Off $=1$

TBS Distance $=33.6298 \quad 50.4466 \quad 66.9889 \quad 70.8256 \quad 60.0197 \quad 40.7397$

Customer $=\begin{array}{llllll}7 & 2 & 3 & 1 & 5 & 4\end{array}$ 
Best TBS $=1$

Next Best TBS $=6$

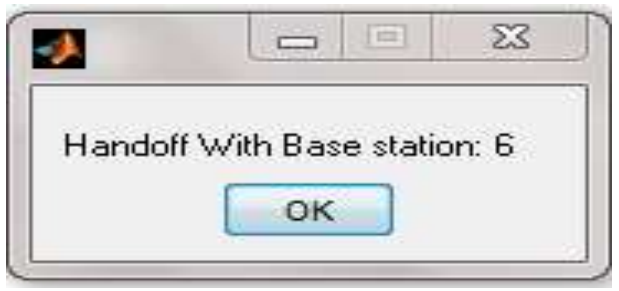

Fig 29 Hand off with base station 6

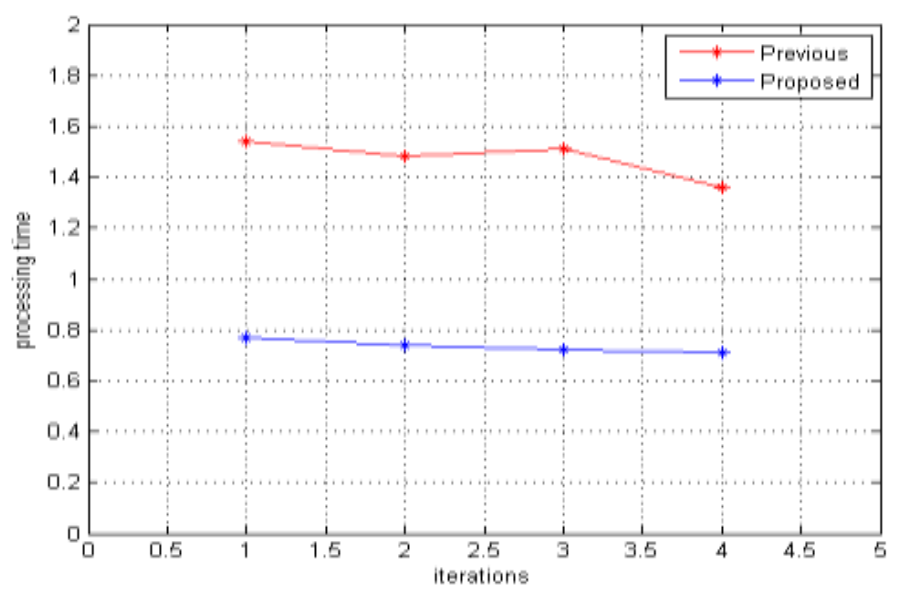

Fig 30 comparison between concurrent and non-concurrent process

The comparison between the delay involved in concurrent and non-concurrent process is shown in figure 30 . From this it is clear that we are able to minimize the scanning delay by an average of $40 \%$.

\section{CONCLUSIONS and FUTURE SCOPE}

\subsection{CONCLUSION}

In case of hand over procedure minimization of pre-hand over delay is an important aspect. By using the MS movement direction prediction (MMDP) based scanning approach the total scanning delay and the scanning interval are reduced. Furthermore on using the concurrent scanning procedure for the best two target BSs we provide high quality hand over support in mobile WiMAX. On performing concurrent scanning procedure the delay involved in scanning TBSs can be reduced by $40 \%$, problems like ideal sectors, network congestion and fast change in RSS are proposed to be minimized.

Efficient routing protocols for the Mobile WiMAX Network are analyzed for best effort and real time application. Fisheye protocol is better to use with small network coverage application. As the network enlarges in size LANMAR gives better performance for real time application. IERP is an efficient routing protocol for the best effort services.

\subsection{FUTURE SCOPE}

In this project node in the target base stations are considered to be at rest. In case of a real time scenario nodes in the target base station are also in motion and there is chances of handover in between the estimated target base station. In this case solution for the network congestion is a challenging task. As a future work analysis of efficient routing protocol for emergency and security application in the Mobile WiMAX environment can be made.

\section{ACKNOWLEDGEMENT}

The Authors would like to thank VGST (Vision Group on Science and Technology), Government of Karnataka, India for providing infrastructure facilities to do this project. Here the authors are made an attempt to reduce the scanning delay by using concurrent scanning process.

\section{REFERENCES}

[1] "Movement Direction-based Handover Scanning for Mobile WiMAX" by Mohammed A.Ben- Mubarak, Borhanuddin Mohd. Ali, Nor K. Noordin, 2011. 17th Asia-Pacific Conference on Communications (APCC) 2nd - 5th October 2011 Sutera Harbour Resort, Kota Kinabalu, Sabah, Malaysia.

[2] WiMAX Forum Certification of Broadband Wireless Systems, www.WiMAXforum.org. 
[3]"Error Control Coding", Shu Lin \& Daniel J. Costello, Jr. Pearson / Prentice Hall, Second Edition, 2004.

[4] Mobile WiMAX Part I: A Technical Overview and Performance Evaluation WiMAX forum 2006.

[5]“Draft IEEE 802.16m System Description Document”, IEEE 802.16 WG, Dec. 2010.

[6] "CTBS: Cost-Effective Target BS Selection Scheme in IEEE 802.16e Networks" Hoon-gyu Choi, Jongpil Jeong, and Hyunseung Choo, 2007 Australasian Telecommunication Networks and Applications Conference December 2nd - 5th 2007, Christchurch, New Zealand.

[7]"Self-Tracking Mobile Station Controls Its Fast Handover in Mobile WiMAX", S. K. Ray, S. K. Ray, K Pawlikowski, A.McInnes and H. Sirisena, IEEE Wireless Communications and Research Conference (WCNC), Sydney, Australia, 18-21 April 2010.

[8]"Resource reservation and allocation based on direction prediction for handoff in mobile multimedia networks", J. Lee, H. Kim, and K. Kim, Computational Science-ICCS 2003, pp. 719-720, 2003.

[9] "Review of Handover to Support Mechanisms Triple Play in Mobile WiMAX", M. Ben-Mubarak, B.M. Ali, N.K. Noordin, A.Ismail and C.K. Ng, IETE Tech Rev, pp. 258-267, 2009. Malaysia.
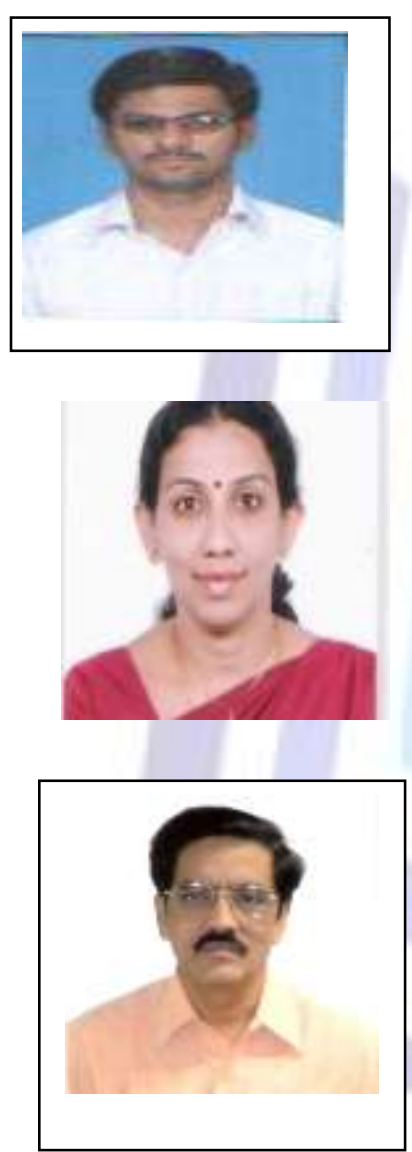

of India, has received six distinguished awards and published over 50 technical papers over support in mobile WiMAX. Communications

and Antennas. INSAT missions.

\section{AUTHORS}

Prabhu Raddy: Received the B.E degree in Electronics and Communication Engineering from Visveswaraya Technological University, Belgaum, India, in 2009. He is currently pursuing $\mathrm{M}$ Tech. in Digital Communication at BMS College of Engineering, Bangalore, India. His research interest include Wireless Communication, QoS for high quality High quality hand

2. Arathi R Shankar: Associate Professor (PG studies)in the Department of Electronics and Communication at BMS College of Engineering, Bangalore India. She has received her BE (Telecommunication) and ME (Electronics) Degree from Bangalore University. She is currently pursuing her PhD from Visveswaraya Technological University, Karnataka, India. She has 21 years of teaching experience and her research interests include Wireless

3. Dr. V. Sambasiva Rao: Professor in ECE Department of PES Institute of technology, Bangalore is an engineering graduate (1973) from College of Engineering, Kakinada, (Andhra University) and obtained Ph.D from BITS, Pilani in 2010. For over 37 years ( April 1974 to June 2011), he was associated with ISRO in various capacities and primarily responsible for the development of high bit rate data transmitters for all IRS series of satellites and various RF and microwave systems in S, C, X, Ku and Ka bands for IRS and

$\mathrm{He}$ has successfully carried out numerous responsibilities as Project Manager/Deputy Project Director for different satellite projects and Deputy Director of Digital\& Communication Area in ISRO Satellite Centre. He is also responsible for various studies related to satellite technologies. Dr. Sambasiva Rao, a Fellow of IETE and a Member of Astronautically Society 